\title{
Numerical Simulation of Heat Removal from Low Dimensional Nanostructures
}

\author{
V. V. MITIN, N. A. BANNOV, R. MICKEVIČIUS and G. PAULAVIČIUS
}

Department of Electrical and Computer Engineering, Wayne State University, Detroit, MI 48202

\begin{abstract}
The acoustic phonon radiation patterns and acoustic phonon spectra due to electron - acoustic phonon interaction in double barrier quantum well have been investigated by solving both the kinetic equations for electrons and phonons. The acoustic phonon radiation patterns have strongly pronounced maximum in the directions close to the perpendicular to the quantum well direction. The radiation pattern anisotropy is explained in terms of possible electron transitions, nonequilibrium electron distribution function, and the Hamiltonian of electron-phonon interactions.
\end{abstract}

Keywords: Phonon-electron Interaction in Quantum Wells, Heat dissipation, Monte Carlo Method, Quantum Kinetic Equation.

\section{INTRODUCTION}

Hot acoustic phonon emission represents one of the major channels for thermal energy removal from heterostructures; in addition, detection of phonons emitted by hot electrons provides a valuable tool for investigation of electron-phonon interactions in heterostructures $[1,2]$. The problem of the spectrum and radiation pattern for acoustic phonons emitted from low dimensional structures has been studied for both quantum wells (QWs) [3,4] and quantum wires [5]. We have investigated the acoustic phonon emission by hot electrons in double barrier heterostructures allowing for electron heating and stimulated phonon emission processes (in previous works [2,3] the electrons were assumed to be at quasiequilibrium). We have solved the electron kinetic equation to obtain the electron distribution function, which has been used to determine the radiation pattern and spectrum of emitted acoustic phonons. The radiation and absorption patterns have highly pronounced maxima inside the solid angle close to the normal to the quantum well direction (z-direction). These orientational dependencies are related to the quantum confinement of electrons, uncertainty in the conservation of the phonon $\mathrm{z}$ component of the wave vector, a peculiar angular dependence of the density of final electron states, and the shape of the electron distribution function.

\section{FORMULATION OF THE PROBLEM}

We will consider a double barrier heterostructure quantum well of width $a$ bounded by planes $z=a / 2$ and $z=-a / 2$. The Cartesian coordinates $x$ and $y$ refer to the plane of the quantum well, the axis $x$ is going in the direction of the average electron velocity (i.e., opposite to the electric field). The axis $y$ augments $x$ and $z$ to a right-handed basis. The dimensions of the quantum well in the $x-y$ plane are $L_{x}$ and $L_{y}$. The 
acoustic phonons are detected by sensors located at a surface of the substrate. We assume, that the distance $d$ from the quantum well to the surface is large in comparison with the lateral dimensions of the quantum well: $d \gg L_{x}, L_{y}$, therefore, the quantum well represents a point source of acoustic phonons. The described geometrical configuration corresponds to experimental setup in Refs. [1,2].

The experimentally measurable quantity is the differential energy flux $\mathcal{G}(\hbar \omega, \Omega)$ (the phonon energy radiated per unit time, per unit solid angle, per unit energy interval), determined by the formula

$$
\mathcal{G}(\hbar \omega, \Omega)=\frac{1}{8 \pi^{3}} \int_{S_{p h-e l}} d \mathbf{S} \frac{\mathbf{q}}{q} u q^{3} N_{\mathbf{q}} .
$$

We will measure it in the units (ps srad) ${ }^{-1}$. In numerical Monte-Carlo calculations, it is convenient to normalize it per one electron. The above integral is taken over a surface, $S_{p h-e l}$, which encloses the quantum well and is discussed in more detail below, $u$ is the sound velocity, $N_{q}$ is the phonon ocupation number, $\Omega$ is the solid angle, $\hbar \omega$ is the phonon energy. Because the differential energy flux is a three dimensional function, it is more convenient to deal with its integrals. The radiation pattern and the radiation spectrum are defined as

$$
\begin{aligned}
\mathcal{G}_{h \omega}(\Omega)=\int_{0}^{\infty} \mathcal{G}(h \omega, \Omega) d(h \omega), \\
\mathcal{G}_{\Omega}(h \omega)=\int_{4 \pi} \mathcal{G}(h \omega, \Omega) d \Omega,
\end{aligned}
$$

respectively.

The differential energy flux has been calculated by integrating the quantum kinetic equation for phonon distribution function over a volume $V_{p h-e l}$, which is bounded by the surface $S_{p h-e l}$. This surface is chosen in such a way that it surrounds the quantum well and is offset by distance $\Delta r$ from it, where $\Delta r$ is the length of the phonon wave packets, $\Delta r \gg a$. Additional assumptions which were made to derive $\mathcal{G}(\hbar \omega, \Omega)$ are the following. The in-plane sizes of the quantum well $L_{x}, L_{y}$ are large enough, $L_{x}, L_{y} \gg \Delta r$, so the 2D electron gas may be treated as homogeneous and the fringe effects may be neglected. The electron lateral confinement is strong, therefore, either only the low- est electron subband is populated or the rate of intersubband transitions is much larger than the rate of intersubband transitions. Under these conditions the electron density matrix is diagonal and corresponds to quasi classical distribution function $f_{\mathbf{k}_{\|}, \mathbf{n}}$. The rate of nonequilibrium phonon emission is assumed to be not very high, so the stimulated phonon emission is proportional to the equilibrium Plank function $N_{h_{w}}$. The deformation potential makes the major contribution to the electron scattering by acoustic phonons. Under these conditions the differential energy flux has the following form

$$
\begin{array}{r}
\mathcal{G}(\hbar \omega, \Omega)=\frac{E_{a}^{2} q^{4}}{4 \pi^{2} \rho u} \sum_{\mathbf{k}_{\|}, n, n^{\prime}} I\left(n^{\prime}, n, q_{z}\right) \times \\
{\left[\left(1-f_{n, \mathbf{k}_{\|}}\right) f_{n^{\prime}, \mathbf{k}_{\|}+\mathbf{q}_{\|}}+\left(f_{n^{\prime}, \mathbf{k}_{\|}+\mathbf{q}_{\|}}-f_{n, \mathbf{k}_{\|}}\right) N_{\hbar \omega}^{T}\right]} \\
\delta\left(\varepsilon_{\mathbf{n}, \mathbf{k}_{\|}}-\varepsilon_{\mathbf{n}^{\prime}, \mathbf{k}_{\|}+\mathbf{q}_{\|}}+\hbar \omega_{q}\right),
\end{array}
$$

where $E_{a}$ is the acoustic deformation potential constant, $\rho$ is the crystal density, $\varepsilon_{n, k_{\|}}$and $\hbar \omega_{q}$ are the electron and phonon energies respectively, $I\left(n^{\prime}, n, q_{z}\right)$ is the overlap integral for electron wave functions in subbands $n$ and $n^{\prime}$ and the phonon plane wave. It is worth mentioning, that Eq. (1) takes into account both the phonon emission and phonon absorption processes. In addition, due to integration over the closed surface $S_{p h-e l}$ which bounds the volume of acoustic phonon interaction with quasi two-dimensional electrons, Eq. (1) gives the differential energy flux in excess of the equilibrium differential energy flux, determined by the function $N_{h_{w}}$.

Outside the volume $V_{p h-e}$ the phonon kinetic equation has the form

$$
\frac{\partial N_{\mathbf{q}}(\mathbf{r})}{\partial t}+\mathbf{u}_{\mathbf{q}} \frac{\partial N_{\mathbf{q}}(\mathbf{r})}{\partial \mathbf{r}}=J_{p h-p h}
$$

where $J_{p h-p h}$ is the integral of phonon - phonon interaction. Because $J_{p h-p h}$ is small over the distance $d$ from the quantum well to a phonon detector (it can be shown by a simple estimate), the phonon propagation may be treated as ballistic and approximation of the geometrical optics can be employed. Therefore, the phonon energy detected by a sensor on a surface of the sample is the same as that determined by Eq. (1). 


\section{RESULTS OF NUMERICAL SIMULATION AND DISCUSSION}

The kinetic equation for electrons takes into account all significant mechanisms of electron scattering in QW: electron scattering by acoustic phonons, as well as electron scattering by confined and interface optical phonons. We have solved the kinetic equation for electrons and obtained the electron distribution function having employed the Monte Carlo technique. The obtained electron distribution function has been used to calculate the differential energy flux (1). However, because $\mathcal{G}(\hbar \omega, \Omega)$ is a three-dimensional function of the spherical angles $\theta, \phi$, and the phonon energy $\hbar \omega$, all results will be presented for integrated over energies or solid angles functions $G_{\hbar \omega}(\Omega)$ and $G_{\Omega}(\hbar \omega)$ respectively.

The radiation patterns of quasi two-dimensional electron gas in electric fields 10,100 , and $1000 \mathrm{~V} / \mathrm{cm}$ are shown in Figs. 1(a), (b), and (c) respectively. To visualize the two-dimensional function $\mathcal{G}_{\hbar \omega}(\Omega)=$ $\mathcal{G}_{\hbar \omega}(\theta, \phi)$, we fix an angle $\phi$ and plot a parametric curve $\left(\left|G_{\hbar \omega}(\theta, \phi)\right| \sin \theta, \mathcal{G}_{\hbar \omega}(\theta, \phi) \cos \theta\right), 0<\theta<\pi / 2$; $\theta=0$ corresponds to the $\mathrm{z}$-direction, $\theta=\pi / 2$ corresponds to a direction in the $x-y$ plane, namely the direction given by the unit vector $(\cos \phi, \sin \phi, 0)$. The plots above the abscissa correspond to prevailing phonon emission (positive $\mathcal{G}_{\hbar \omega}(\theta, \phi)$ ), the plots below the abscissa correspond to prevailing phonon absorption (negative $\mathcal{G}_{\hbar \omega}(\theta, \phi)$ ). The spectra of the acoustic phonons given by the function $G_{\Omega}(\hbar \omega)$ is shown on Fig. 2 for several electric fields.

For a given $\phi$, electrons can scatter in two regions in the $\mathbf{k}_{\|}$-space. It gives rise to two peaks of function $\mathcal{G}_{\hbar \omega}(\theta, \phi)$ for two different $\theta$ (similar to forward and backward scattering in 1D case [5]). These peaks are very sharp in terms of $\theta$ variable. However, if the electron distribution function is close to equilibrium as in Fig. 1 (a), these peks are completely smeared due to averaging over electron distribution function. In stronger fields electron distribution function has streaming-like character. For this reason the the radiation patterns become more anisotropic (see Figs. 1 (b) and (c)).
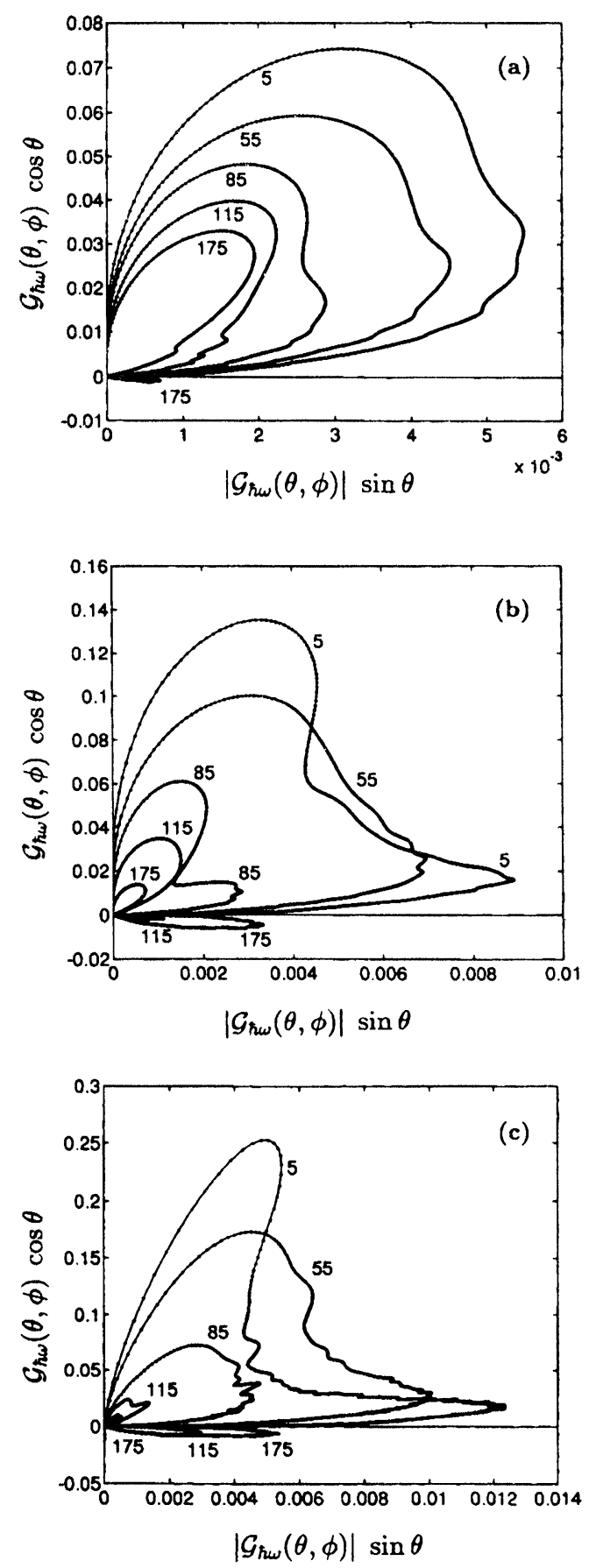

FIGURE 1 Radiation pattern of acoustic phonons, $\mathcal{G}_{\hbar \omega}(\Omega)$, in units $\mathrm{meV} /$ (ps srad electron) for azimuthal angles $\phi=5^{\circ}, 55^{\circ}$, $85^{\circ}, 135^{\circ}, 175^{\circ}$. The numbers at the curves correspond to the angles $\phi$. GaAs/AlAs quantum well of width $100 \AA$, lattice temperature $T=30 K$. Electric field $10 \mathrm{~V} / \mathrm{cm} \mathrm{(a),} 100 \mathrm{~V} / \mathrm{cm}$ (b), $1000 \mathrm{~V} / \mathrm{cm}(\mathrm{c})$ 


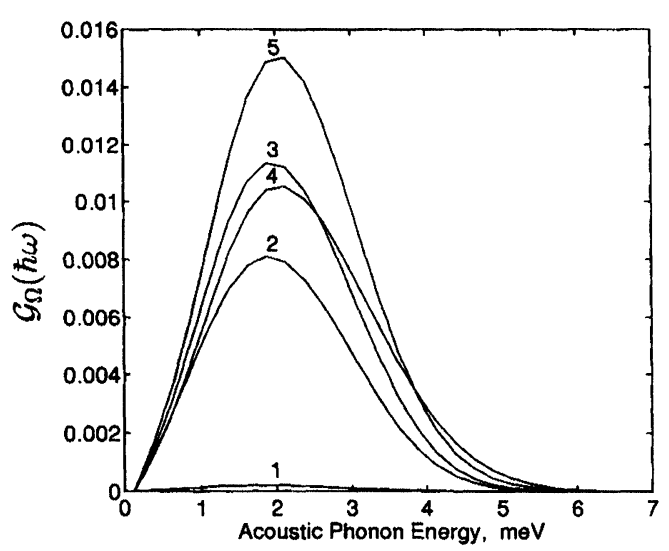

FIGURE 2 The spectra of the acoustic phonon energy flux, $\mathcal{G}_{\Omega}(\omega)$, in units $1 /$ (ps electron) for electric fields $1 \mathrm{~V} / \mathrm{cm}(1), 10 \mathrm{~V} / \mathrm{cm}(2)$, $100 \mathrm{~V} / \mathrm{cm} \mathrm{(3),} 300 \mathrm{~V} / \mathrm{cm}(4)$, and $1000 \mathrm{~V} / \mathrm{cm}$ (5)

In spite of the significant modification of the phonon radiation patterns when the electric field grows, the spectrum of irradiated phonons remains remarkably unchanging (see Fig. 2). The average energy of the phonons is determined by the width of the quantum well and is approximately equal to $2 \pi \hbar u / a$. The position of the maxima may be changed only if both the lattice temperature and the electric field are so low, that the average electron energy is smaller than $2 \pi \hbar u / a$. Eq. (1) gives the differential phonon energy flux in excess of the thermal equilibrium background determined by $N_{\hbar \omega}^{T}$. Therefore, the total energy of the radiated phonons is equal to zero if $E=0$. It grows in small electric fields and then becomes almost field independent. It happens because the average phonon energy does not depend on the electric field, and the electron-acoustic phonon scattering rate in one electron subband in 2D case is also almost constant due to energy independent density of states.

\section{Acknowledgements}

This work was supported by ARO.

\section{References}

[1] J. K. Wigmore, M. Erol, M. Sahraoui-Tahar, M. Ari, C. Wilkinson, J. Daviest, M. Holland, C. Stanley, Measurement of energy-loss from a HEMT structure by observing emitted phonons, Semicond. Sci. Technol. 8, 322 (1993).

[2] M. Ashe, R. Heg, H. Kostial, B. Danilchenko, A. Klimashov, S. Roshko, Hot electrons and nonequilibrium phonons in a multiple $\delta$-layer-doped GaAs structure, Phys. Rev. B, 51, 7966 (1995).

[3] F. T. Vasko, Emission of acoustic phonons by two-dimensional electrons, Sov. Phys. Solid State 30, 1207 (1989).

[4] W. Xu, J. Mahanty, Acoustic phonon emission angle in selectively doped AlGaAs-GaAs-AlGaAs single quantum well, J. Phys. : Condens.Matter 6, 6265 (1994).

[5] R. Mickevicius, V. Mitin, V. Kochelap, M. A. Stroscio, G. J. Iafrate, Radiation of acoustic phonons from quantum wires, J. Appl. Phys. 77, 5095 (1995). 

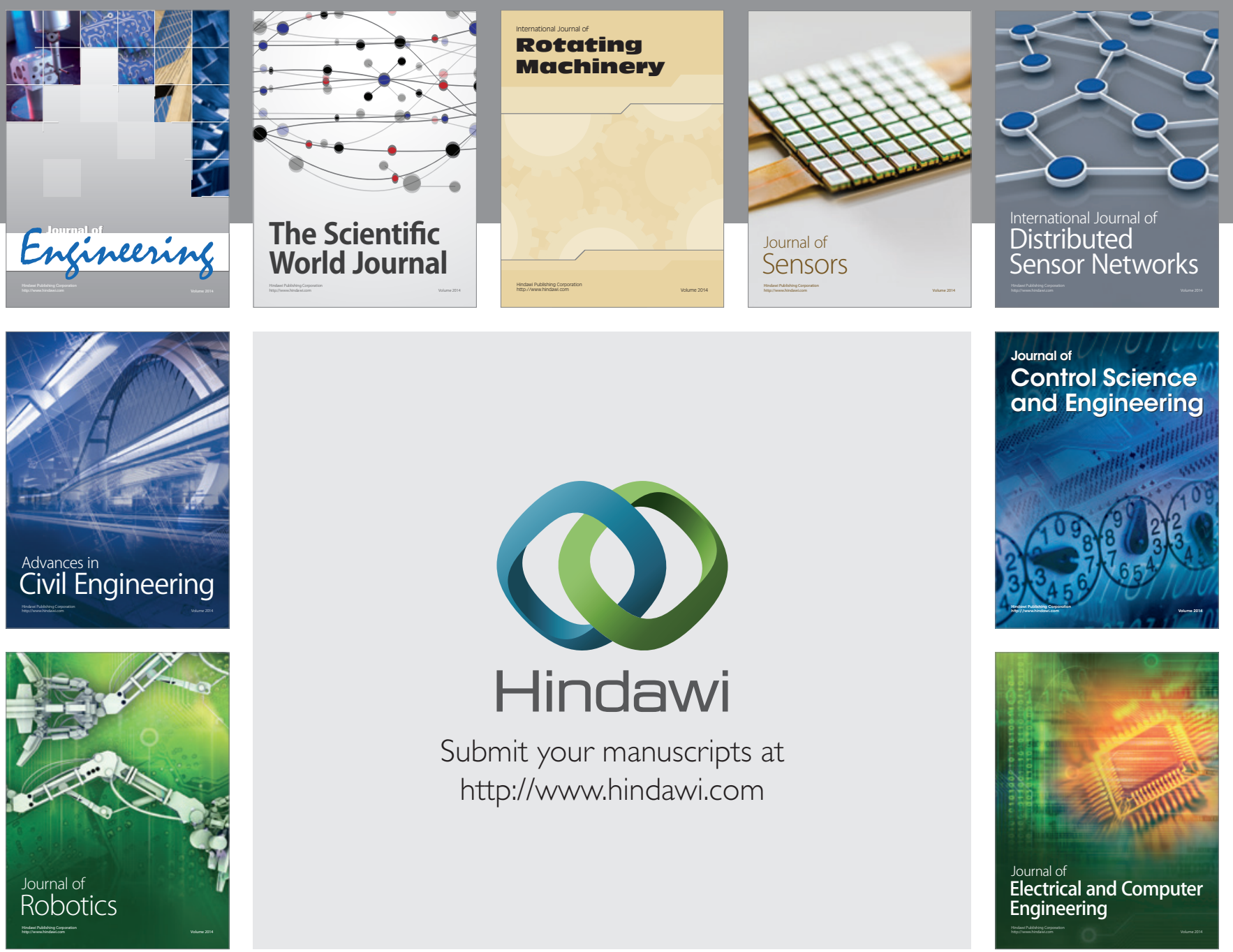

Submit your manuscripts at

http://www.hindawi.com
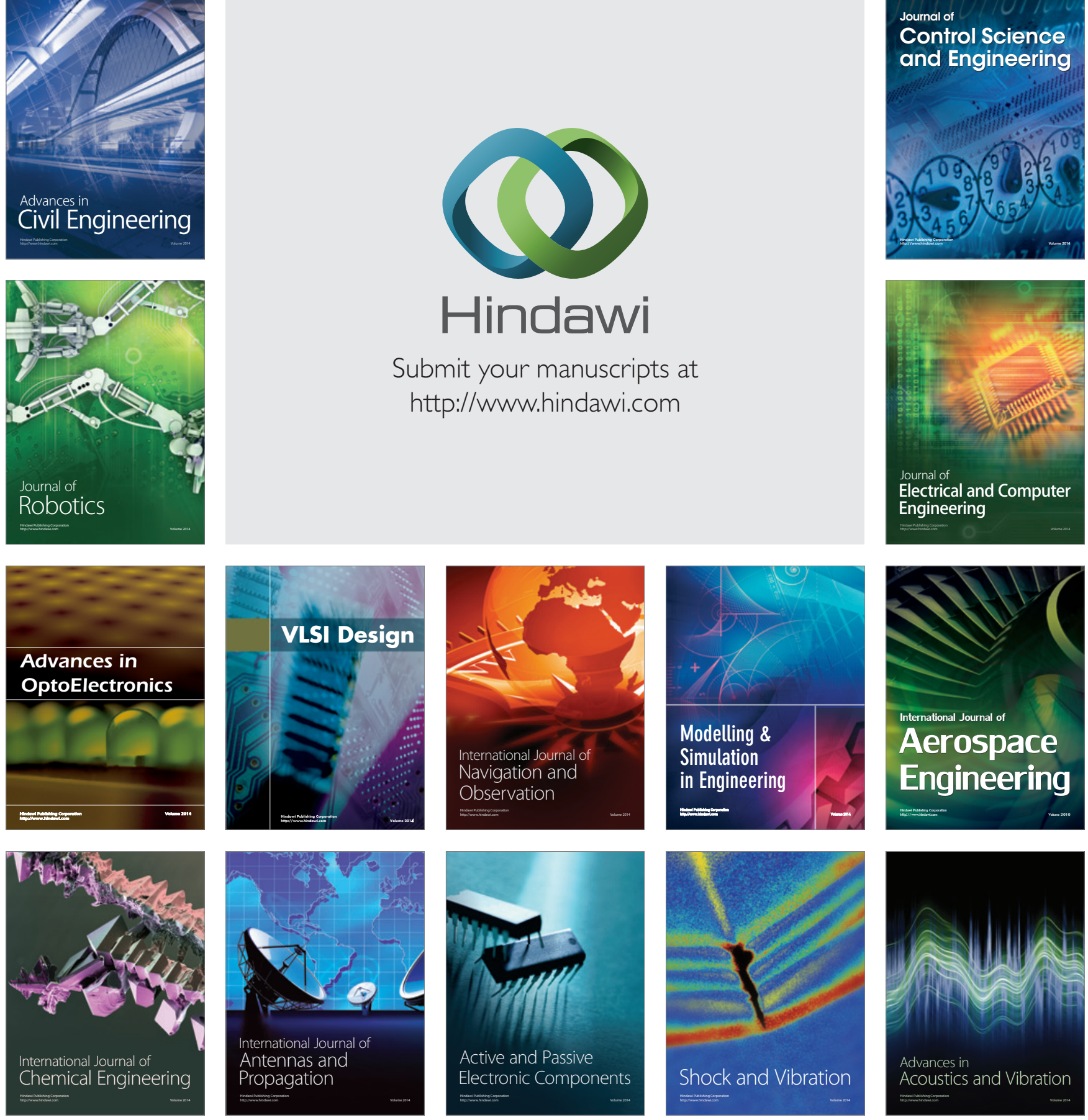